ARTICLE

Received 26 Feb 2015 | Accepted 15 Jul 2015 | Published 25 Aug 2015

DOI: 10.1038 /ncomms 9098

OPEN

\title{
Atomically controlled substitutional boron-doping of graphene nanoribbons
}

\author{
Shigeki Kawai ${ }^{1,2}$, Shohei Saito ${ }^{2,3}$, Shinichiro Osumi ${ }^{3}$, Shigehiro Yamaguchi ${ }^{3,4,5}$, Adam S. Foster ${ }^{6}$, Peter Spijker ${ }^{6}$ \\ \& Ernst Meyer ${ }^{1}$
}

Boron is a unique element in terms of electron deficiency and Lewis acidity. Incorporation of boron atoms into an aromatic carbon framework offers a wide variety of functionality. However, the intrinsic instability of organoboron compounds against moisture and oxygen has delayed the development. Here, we present boron-doped graphene nanoribbons (B-GNRs) of widths of $N=7,14$ and 21 by on-surface chemical reactions with an employed organoboron precursor. The location of the boron dopant is well defined in the centre of the B-GNR, corresponding to 4.8 atom\%, as programmed. The chemical reactivity of B-GNRs is probed by the adsorption of nitric oxide (NO), which is most effectively trapped by the boron sites, demonstrating the Lewis acid character. Structural properties and the chemical nature of the NO-reacted B-GNR are determined by a combination of scanning tunnelling microscopy, high-resolution atomic force microscopy with a $\mathrm{CO}$ tip, and density functional and classical computations.

\footnotetext{
${ }^{1}$ Department of Physics, University of Basel, Klingelbergstrasse 82, CH-4056 Basel, Switzerland. ${ }^{2}$ PRESTO, Japan Science and Technology Agency, 4-1-8, Honcho, Kawaguchi, Saitama 332-0012, Japan. ${ }^{3}$ Department of Chemistry, Graduate School of Science, Nagoya University, Furo, Chikusa, Nagoya 464-8602, Japan. ${ }^{4}$ Institute of Transformative Bio-molecules, Nagoya University, Furo, Chikusa, Nagoya 464-8602, Japan. ${ }^{5}$ CREST, Japan Science and Technology Agency, 4-1-8, Honcho, Kawaguchi, Saitama 332-0012, Japan. ${ }^{6}$ COMP, Department of Applied Physics, Aalto University, PO Box 11100, FI-00076 Helsinki, Finland. Correspondence and requests for materials should be addressed to S.K. (email: shigeki.kawai@unibas.ch).
} 
oron is a unique element in terms of electron deficiency and Lewis acidity, yet it has a comparable size to carbon. Incorporation of boron atoms into an aromatic carbon framework like carbon nanotubes ${ }^{1}$ and graphene ${ }^{2}$ offers a wide variety of functionality in, for example, chemical sensing 3,4 , nanoelectronics ${ }^{5,6}$, photocatalysis ${ }^{7}$ and battery electrodes ${ }^{8}$. So far, the syntheses have been demonstrated by both the chemical vapour deposition method with co- or mono-depositing of small molecules, like $\mathrm{B}_{2} \mathrm{H}_{6}, \mathrm{BCl}_{3}$, and $\mathrm{PhB}(\mathrm{OH})_{2}$ (refs 7-11), and by the reactive microwave plasma method with $\mathrm{B}\left(\mathrm{CH}_{3}\right)_{3}$ (ref. 12). However, besides the formation of Stone-Wales defects, ${ }^{10}$ the coordination environment of the boron sites in the graphitic carbons, namely the doping site and density of boron, cannot be precisely controlled in these methods. This limitation can be overcome by a recently developed on-surface chemical reaction ${ }^{13,14}$. In fact, nitrogen atoms have been doped into the edges of graphene nanoribbons (GNRs) by employing nitrogen-doped precursor molecules ${ }^{15,16}$. In contrast, the intrinsic instability of organoboron compounds against moisture and oxygen has delayed the development of boron-doped nanocarbon chemistry ${ }^{17,18}$ and has led to the unavailability of boron-doped precursor molecules. However, our recent chemical synthesis of polycyclic aromatic hydrocarbons with boron atoms proved the stability of this materials class ${ }^{19}$. The use of such an organoboron precursor can pave the way for boron doping into GNRs. We now present a well-structured boron-doped-GNR (B-GNR) in terms of valency, position and doping ratio and study its chemical structure and property by high-resolution atomic force microscopy (AFM) and scanning tunneling microscopy (STM).

\section{Results}

Synthesis of boron-doped graphene nanoribbons. Figure 1a shows the chemical structure of the precursor molecule, in which two pristine anthracene units and one 9,10-dibora-9,10dihydroanthracene moiety are covalently bonded while two bromine atoms are located at both termini. By annealing the molecules on a $\mathrm{Au}$ surface at $180^{\circ} \mathrm{C}$, the bromine atoms are removed by surface catalytic dehalogenation. Consequently, the precursor molecules are linearly polymerized. At an elevated temperature of up to $400^{\circ} \mathrm{C}$, the surface-assisted cyclodehydrogenation results in the formation of an aromatic graphene nanoribbon ${ }^{20}$. Employing this reaction, boron atoms can be doped as $\mathrm{BC}_{3}$ Lewis acidic sites at the centre row in the armchair-edge GNR (B-GNR) with a width of seven carbon atoms $(N=7)$. The interval between the boron sites is fixed with a doping density of 4.8 atom\%.

In experiments, the precursor molecules were deposited on a clean $\mathrm{Au}(111)$ surface in ultra-high vacuum and were subsequently annealed at $180^{\circ} \mathrm{C}$ (Fig. 1b). At $4.8 \mathrm{~K}$ the molecules mainly stay along the valley of the herringbone structure on $\mathrm{Au}(111)$. We performed tip-induced lateral manipulation to check the presence of the polymer chain. After scanning the tip at a constant height along the line indicated with an arrow in Fig. 1b, several molecular units moved together, indicating successful polymerization (Fig. 1c). The successive anthracene units have opposite tilts to the Au substrate owing to the repulsion between hydrogen atoms in the adjacent anthracene moieties. After annealing at $400^{\circ} \mathrm{C}$, such corrugated structures disappeared. Instead, stripe-shaped flat nanoribbons appeared (Fig. 1d).

The observed STM contrast is inverted by the polarity of the applied tip bias voltage ( -3 and $3 \mathrm{~V}$ ), indicating the perturbation of the electronic structure by the doped boron atoms as an electron-accepting unit (Fig. 1e,f, Supplementary Fig. 1). Pink crosses indicate the same atomic site in B-GNR. By setting close to zero bias voltage $(-2 \mathrm{mV})$, the electronic states near the Fermi level of B-GNR are resolved (Fig. 1g). We also observed two different contrasts at the armchair type edge, where the ratio of

a
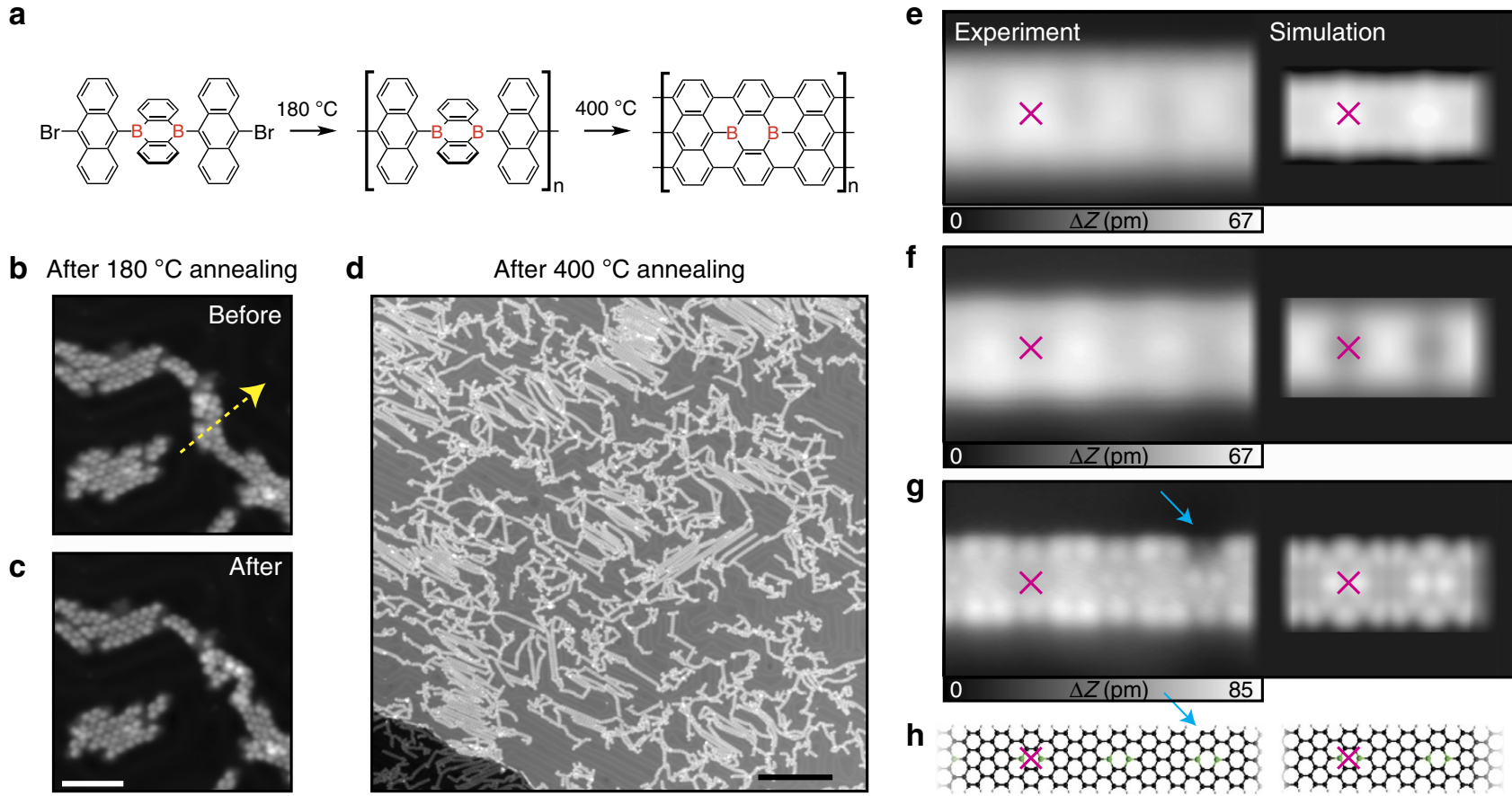

$\mathbf{f}$

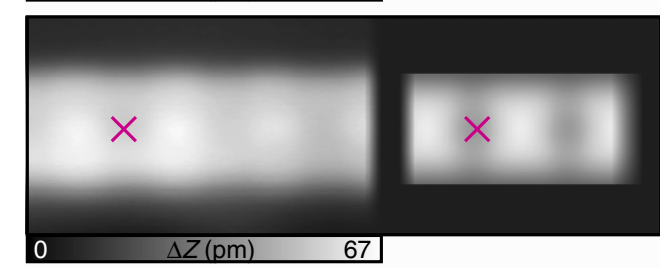

g

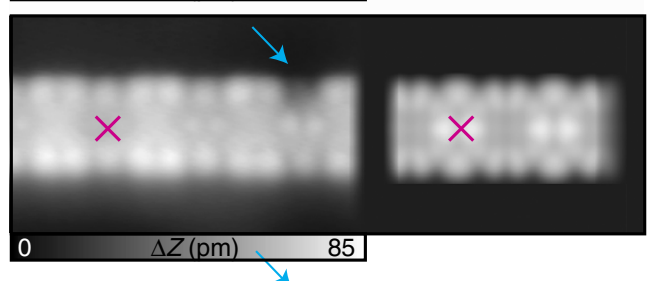

h

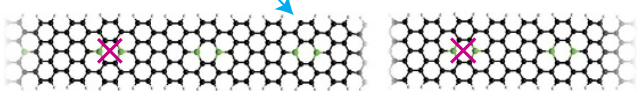

Figure 1 | Synthesis of boron-doped graphene nanoribbons. (a) Schematic drawing of the on-surface chemical reaction. (b) STM of molecules, polymerized by annealing at $180^{\circ} \mathrm{C}$ and (c) after the tip-induced manipulation. Scale bar, $5 \mathrm{~nm}$. (d) B-GNR, synthesized by annealing at $400{ }^{\circ} \mathrm{C}$. Scale bar, $30 \mathrm{~nm}$. (e-g) High-resolution STM topographies (left) and corresponding simulated STM images (right) of B-GNR, taken with different bias voltages. (h) Chemical structure of B-GNR. Black and pink balls indicate carbon and boron atoms. Measurement parameters: tunnelling current $I=5 \mathrm{pA}$ and bias voltage applied to the tip $V=-200 \mathrm{mV}$ for $(\mathbf{b}-\mathbf{d}), I=10 \mathrm{pA}$ and $V=-3 \vee$ for $(\mathbf{e}), I=10 \mathrm{pA}$ and $V=3 \vee$ for $(\mathbf{f})$, and $I=1 \mathrm{nA}$ and $V=-2 \mathrm{mV}$ for $(\mathbf{g})$. 
one dark- and two bright-contrasts suggests that the darkcontrast edge relates to the boron-doped anthracene. The hydrogen atoms at the armchair edge can be desorbed by the tunnelling current at a large negative tip bias $(-3.5 \mathrm{~V}$ and 100 pA, Supplementary Fig. 2) as indicated with an arrow. The simulated STM topographies (right panel in Fig. 1e-g) are in good agreement with the experimental results (left panel), so that the chemical structure can be unambiguously assigned as shown in Fig. 1h.

Electronic structure of boron-doped graphene nanoribbons. To measure the electronic structure of $N=7$ B-GNR, $\mathrm{d} I / \mathrm{d} V$ spectra were taken with a clean Au tip above four different sites of $\mathrm{B}-\mathrm{GNR}$ and, for reference, above one $\mathrm{Au}(111)$ site (Fig. 2a). The conductance is related to the local density of states at the tip position. At the $\mathrm{Au}(111)$ site, the effect of Shockley surface states locates around $-0.5 \mathrm{eV}$ below the Fermi energy. The contribution, originating from the surface state, is even visible on B-GNR as modulations of the $\mathrm{d} I / \mathrm{d} V$ spectrum down to $-1.0 \mathrm{eV}$, and rather stronger than on the non-doped $\mathrm{GNR}^{21-23}$. This may relate to the stronger coupling to the substrate due to the presence of boron atoms. More interestingly, in contrast to the pristine $\mathrm{GNR}^{22}$, the

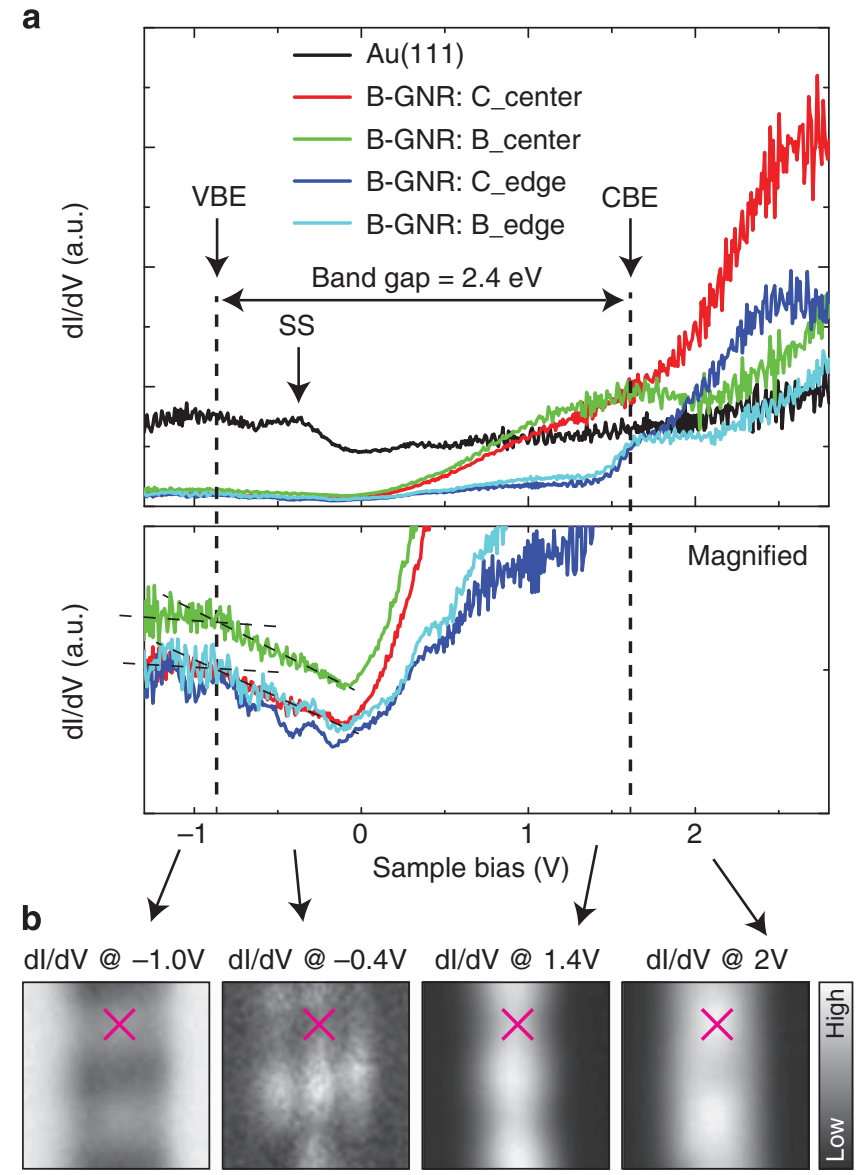

Figure 2 | $\mathbf{d} \boldsymbol{I} / \mathbf{d} \boldsymbol{V}$ curve on B-GNR. (a) Differential conductance ( $d / / d V$ ) spectra taken at four different sites of $N=7$ B-GNR and one Au(111) site with a clean Au tip. The spectra were numerically calculated from the measured bias dependent curves of the tunnelling current. The bias voltage is only here redefined with respect to the tip grounding instead of the sample grounding. (b) Constant height $\mathrm{d} l / \mathrm{d} V$ maps measured with a lock-in amplifier (root mean square amplitude $=14 \mathrm{mV}$ and frequency $=521 \mathrm{~Hz}$ ) at the different bias voltages. Pink crosses indicate at the centre of the same $\mathrm{C}_{4} \mathrm{~B}_{2}$ site. presence of these boron atoms downshifts the interface state. Nevertheless, the valence band edge locates around $-0.8 \mathrm{eV}$ below the Fermi energy, with clear peaks at $1.6 \mathrm{eV}$ corresponding to the conduction band edge. Consequently, the band gap of the B-GNR is $2.4 \mathrm{eV}$, which is comparable to the non-doped GNR on $\mathrm{Au}(111)$ (ref. 22). Previous theoretical studies indicate that the position of doped boron strongly affects the modulation of the electronic structure and so that the minimum (maximum) effect is expected with a doping at the centre (edge $)^{5}$. In this system, boron atoms locate exactly at the centre of $N=7$ B-GNR and, thus, no significant electron-hole asymmetry is present (full suppression of backscattering), leaving intact the overall electronic structure (this is seen also in our calculated electronic structure, Supplementary Fig. 3). Yet, in a detailed inspection, the presence of boron atom in the GNR can be observed. The inversions of the conductance between the doping and non-doping sites occur twice between 1.4 and $2.0 \mathrm{eV}$ and between -1.0 and $-0.4 \mathrm{eV}$ both at the edge and centre. This is consistent with the scanning tunnelling spectroscopy (STS) images taken within the energy levels at constant heights (Fig. 2b), and also the location of the boron features in the calculated projected density of states.

Fused boron-doped graphene nanoribbons. After the substrate was further annealed at $510^{\circ} \mathrm{C}$, B-GNRs with different widths $(N=7,14$ and 21) were obtained by fusing the armchair edges (Fig. 3a). To check the chemical structure in detail, we used an AFM with a CO-functionalized tip ${ }^{24}$. In this technique, when the tip-sample separation is small enough, the tip detects the short-range Pauli repulsive interaction ${ }^{25}$ and the bond structure of molecules can be resolved via the frequency shift of the oscillating force sensor ${ }^{24,26-28}$. Figure $3 \mathrm{~b}$ shows the AFM image of $N=7$ B-GNR obtained in a constant height mode. The
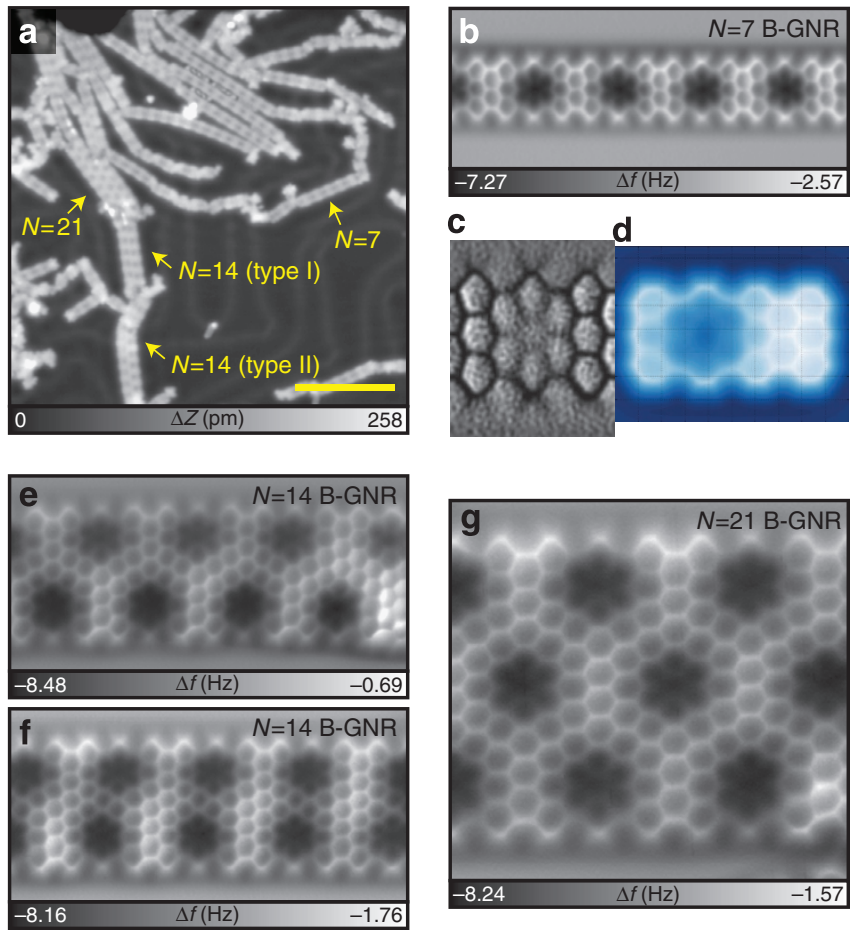

Figure 3 | Fused B-GNR. (a) STM overview of fused B-GNR. Scale bar, $10 \mathrm{~nm}$. (b) Frequency shift $\Delta f$ map of $N=7$ B-GNR and (c) the corresponding Laplace filtered image for a better view of bonds. (d) and the simulated AFM image. $(\mathbf{e}, \mathbf{f}) \Delta f$ maps of fused $N=14$ B-GNR with different structures. (g) $\Delta f$ map of fused $N=21$ B-GNR. Measurement parameters: $A=38 \mathrm{pm}$ and $\mathrm{V}=0 \mathrm{~V}$. 
periodic aromatic carbons are clearly revealed, in which every three anthracene units have a defect-like feature, with darker contrast (more negative frequency shift). To enhance the observed bond visually, a Laplace filter ${ }^{27}$ was applied in the region around the darker contrast site (Fig. 3c). The $\mathrm{C}-\mathrm{C}$ bonds in the anthracene unit are clearly observed and, even at the centre, the B-C bonds were observed.

Although the AFM imaging mechanism with a $\mathrm{CO}$ tip is somewhat sensitive to the total density of charge $e^{25}$, the nature of the contrast cannot fully be explained by the inherent electron-deficient nature of boron and its electron-accepting character. Thus, we consider the topographic geometry in detail. Although the van der Waals radius of the B atom (192 pm) is larger than the $\mathrm{C}$ atom $(170 \mathrm{pm})$, the actual topographic shift of the boron atom core in B-GNR on $\mathrm{Au}$ obtained by our density functional theory (DFT) calculation is a $30 \mathrm{pm}$ dip (Supplementary Fig. 4), which clearly plays an important role in the more negative frequency shift observed and predicted in simulations (see also calculated the AFM image in Fig. 3d and Supplementary Fig. 5). In the absence of this topographic distortion, the difference in local charge density and resulting electrostatic interactions is not enough to produce a clear contrast change. The $\mathrm{B}-\mathrm{C}$ bond (longer than the $\mathrm{C}-\mathrm{C}$ bond) also deforms the honeycomb structure ${ }^{19}$, while the distortion of the carbon ring in the AFM image relates to the effect of tilting CO tip $^{27}$.

On the basis of these observations, we state that the on-surface chemical reaction was accomplished as demonstrated in Fig. 1a. Some defects, presumably relating to the on-surface dissociation of $\mathrm{B}-\mathrm{C}$ bonds in the first annealing process, are also observed (Supplementary Figs 6 and 7). We further analysed B-GNRs with wider widths (Supplementary Fig. 8). For $N=14$ B-GNRs, two different fused configurations with the out-of-phase (Fig. 3e) and the shifted phase (Fig. 3f) were observed. Since the configuration is determined by the relative position in the fusing process, the shifted phase $N=14$ B-GNR has a mirror configuration, thus, in total three configurations. In such a process, the $\mathrm{C}-\mathrm{C}$ link seems to be caused by a zipping up process through dissociating hydrogen atoms at the armchair edge (Supplementary Fig. 9). For $N=21$ B-GNRs, nine different textures are theoretically expected. Among them, the perfectly ordered out-of-phase structure is shown in Fig. 3g. The boron atoms can stay at the designated atomic site throughout the annealing process at the higher temperature of $510^{\circ} \mathrm{C}$. Such high stability may reflect the robustness of the $\mathrm{BC}_{3}$ site ${ }^{29}$. Therefore, once a boron atom is perfectly incorporated in graphene, the thermal stability is remarkably high (Supplementary Fig. 10).

Lewis acidity of the boron site. Since the structural and electronic characters of the $\mathrm{BC}_{3}$ sites were demonstrated, we next evaluate the Lewis acidity of the boron sites by exposing the B-GNRs to nitric oxide (NO) gas. Figure 4a shows a large-scale STM image, where small dots correspond to dosed NO molecules. It is well-known that the NO molecules adsorb at the elbow site of the $\mathrm{Au}(111)$ herringbone structure (blue arrow) ${ }^{30}$. More preferentially, NO molecules attach to the edge of the B-GNR (red arrow). However, it is more important that the NO molecules were most efficiently trapped at the centre of the B-GNR (yellow arrow), corresponding to the boron site. By setting the tip closer to the sample surface, this NO molecule can be easily manipulated by the scanning tip. Figure $4 \mathrm{~b}-\mathrm{d}$ present a series of the STM topographies, showing the movement of NO. The half-moon-like feature in Fig. 4c corresponds to the manipulated NO by the scanning local probe. Since the NO molecule showed a hopping behaviour on specific boron sites, the $\mathrm{BC}_{3}$ moieties exhibit a Lewis acidic role for the
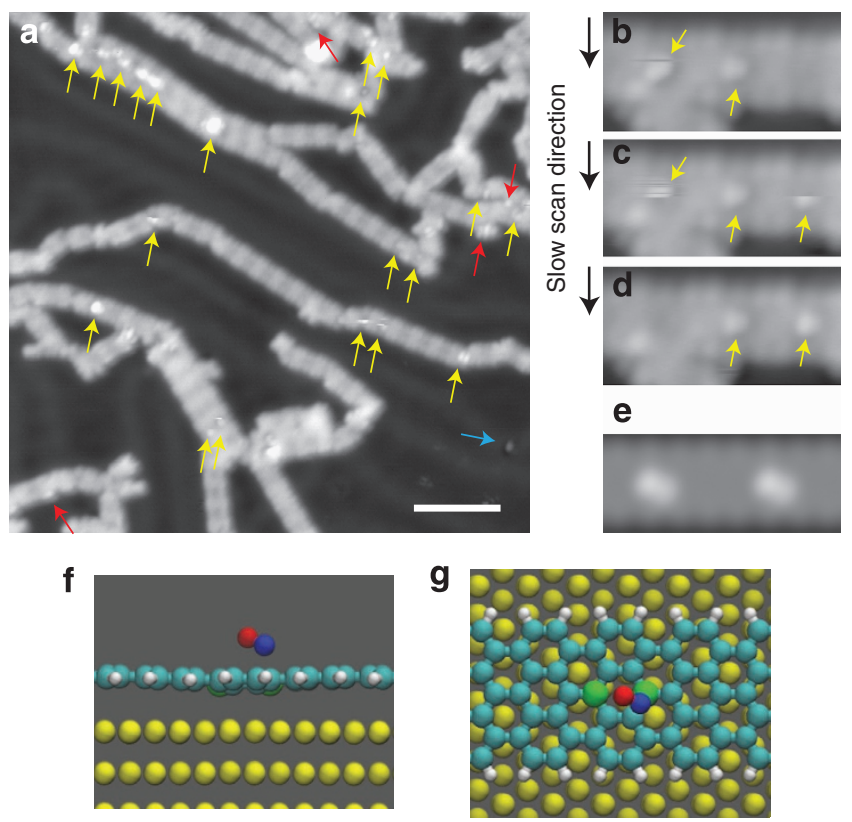

Figure 4 | NO adsorption. (a) STM topography of B-GNR with NO molecules. Blue, red and yellow arrows indicate NO molecules attached at the elbow of herringbone structure on $\mathrm{Au}(111)$, the armchair edge, and the boron site of the B-GNRs, respectively. Scale bar, $5 \mathrm{~nm}$. (b-d) A series of STM images to show the hopping NO between the boron sites. (e) Simulated STM image. $(\mathbf{f}, \mathbf{g})$ Side and top views of calculated conformation of NO adsorbed at the boron site in B-GNR. Nitrogen, oxygen and boron atoms are shown in blue, red and green, respectively. Measurement parameters: $V=-200 \mathrm{mV}$ and $I=1 \mathrm{pA}$ in (a) and $I=2 \mathrm{pA}$ in (b-d).

stable gas adsorption. The simulated STM image (Fig. 4e) with optimized adsorption model (Fig. 4f,g) shows that the NO molecule is bound to the $\mathrm{B}$ site via the $\mathrm{N}$ atom. The calculated binding energy of $1.5 \mathrm{eV}$ is ideal for a chemical sensor, since a continuous adsorption at room temperature is expected to show a linear response to the NO density. The NO chemical adsorption significantly changes the electronic structure of the B-GNR, introducing new states into the band gap (Supplementary Fig. 3), which suggests a strong electric response in a device. To refresh the device and avoid saturation, a small increase in thermal energy will cause desorption.

\section{Discussion}

We present a programmed synthesis of boron-doped graphene nanoribbons with uniform texture of Lewis acidic site. Ullmann type on-surface polymerization and the following thermal cyclodehydrogenation reactions were employed for a triarylborane precursor with an elaborate design. The uniform $\mathrm{BC}_{3}$ sites are doped at designated positions with a fixed doping ratio according to the precursor. Namely, two boron atoms exist at the centre row in every three anthracene moieties and the doping density is 4.8 atom\%. The chemical structures of B-GNRs with different widths of $N=7,14$ and 21 were directly resolved by high-resolution force microscopy with a CO tip. Lewis acidity of the electron-deficient boron sites is experimentally demonstrated by nitric oxide adsorption at thermal equilibrium. This behaviour proposes a new approach for temperature-dependent dynamic perturbation of the electronic properties of B-GNRs around the Fermi level in an NO atmosphere, while the previously reported structural modifications, by varying the width of the pristine $\mathrm{GNR}^{31}$ or by nitrogen atom doping of the edge $\mathrm{e}^{15,16}$, have realized 
only static control. The tunable response presented here offers great potential in many technologies, particularly where dynamic control would be a disruptive development. Besides this application, the fabrication of boron-doped nanoribbons could allow for the design of mobility gaps and novel types of graphene transistors $^{32,33}$. In the $N=7 \mathrm{~B}$-GNR, the boron locates exactly at the centre of the ribbon, so that the backscattering is fully suppressed. Yet, in the fused B-GNRs, the symmetry can vary. Further, the asymmetry can be increased by co-depositing boron-doped and non-doped precursor molecules. To fabricate such devices, transferring on-surface synthesized B-GNRs is a critical issue, yet we believe that the recently developed thin film etching technique can solve this ${ }^{16,34}$.

\section{Methods}

Experimental measurement. All experiments were performed with an Omicron STM/AFM with a qPlus configuration ${ }^{35}$, operating at $4.8 \mathrm{~K}$ in ultrahigh vacuum. A clean $\mathrm{Au}(111)$ surface was in-situ prepared by repeated cycles of standard sputtering and annealing. The tungsten tip of a tuning fork sensor was ex-situ sharpened by a focused ion beam milling technique and was then in-situ covered with $\mathrm{Au}$ atoms by contacting to the sample surface. The resonance frequency of the self-oscillating qPlus sensor was detected by a digital PLL circuit (Nanonis: OC4 and Zurich Instruments: HF2LI and PLL). 9,10-bis(10-bromoanthracen-9-yl)9,10-dihydro-9,10-diboraanthracene was deposited on the substrate from a crucible of Knudsen cell, resistively heated at $240^{\circ} \mathrm{C}$. Subsequently, the sample was annealed at 180 and $400^{\circ} \mathrm{C}$ to synthesize the $N=7$ B-GNR. The fused B-GNRs with $N=14$ and 21 were obtained by further annealing at $510^{\circ} \mathrm{C}$. Further, NO gas was introduced into the ultrahigh vacuum chamber (100 Langmuir) while the sample stayed in the cold microscope. In STM mode, the tip was biased while the sample was electronically grounded. The topographic images were taken in a constant current mode. In AFM mode, the tip apex was terminated by a CO-molecule and all images were taken at a constant height mode.

Synthesis of precursor molecule. The precursor 1, 9,10-bis(10-bromoanthracen9-yl)-9,10-dihydro-9,10-diboraanthracene, was obtained from compounds 2 and 3 through the synthetic route shown in Supplementary Fig. 11a. Although some derivatives of the precursor 1 with extra bulky substituents have been reported ${ }^{36}$, the precursor $\mathbf{1}$ has not been characterized because of its low solubility in common organic solvents. We successfully characterized the isolated precursor $\mathbf{1}$ by the NMR analyses in tetrachloroethane at $100^{\circ} \mathrm{C}$ and by X-ray crystal structure analysis using chlorobenzene as solvent. The crystalline sample of the precursor 1 can be stored at ambient conditions as a result of the steric protection of the boron atoms by the anthracene groups. Compounds 2 (ref. 37) and 3 (ref. 38) were prepared according to literature procedures. The compound $\mathbf{3}$ was stored in a glove box due to the moisture sensitivity. All reactions were carried out under a nitrogen atmosphere. Commercially available solvents and reagents were used as received. Dry ether and $n$-BuLi (1.6 M in hexane) were purchased from Wako Pure Chemical Industries. Dry toluene was purchased from Kanto Chemical.

Precursor molecule 1. To a solution of $2(231 \mathrm{mg}, 0.605 \mathrm{mmol})$ in ether $(13 \mathrm{ml})$ cooled at $0{ }^{\circ} \mathrm{C}$ was added $n$-BuLi $(1.6 \mathrm{M}$ in hexane, $0.378 \mathrm{ml}, 0.605 \mathrm{mmol})$ dropwise. The mixture was stirred at room temperature for $20 \mathrm{~min}$. After removing the solvent in vacuo, the residue was dissolved together with 3 (101 mg, $0.303 \mathrm{mmol})$ in toluene $(8 \mathrm{ml})$. The resulting mixture was stirred at room temperature for $16 \mathrm{~h}$, and the solvent was removed in vacuo. The crude product was washed with $\mathrm{MeOH}$ and hexane and further purified by silica gel column chromatography using $\mathrm{CHCl}_{3} /$ hexane (ratio 2:1) as an eluent. Recrystallization from chlorobenzene afforded 1 as an orange solid $(31.2 \mathrm{mg}, 45.5 \mu \mathrm{mol}, 15 \%)$. Thin layer chromatography $\left(\mathrm{CHCl}_{3} /\right.$ hexane, $\left.2: 1 \mathrm{v} / \mathrm{v}\right): R_{\mathrm{f}}=0.90 ;{ }^{1} \mathrm{H}-\mathrm{NMR}$ $\left(600 \mathrm{MHz}, 1,1,2,2\right.$-tetrachloroethane- $\left.d_{2}, 100^{\circ} \mathrm{C}\right): \delta 8.72 \mathrm{ppm}(\mathrm{d}, J=9.0 \mathrm{~Hz}, 1 \mathrm{H})$, $7.77(\mathrm{~d}, J=8.4 \mathrm{~Hz}, 1 \mathrm{H}), 7.68\left(\mathrm{dd}, J_{1}=9.0 \mathrm{~Hz}, J_{2}=7.8 \mathrm{~Hz}, 1 \mathrm{H}\right), 7.46(\mathrm{~m}, 1 \mathrm{H}), 7.43$ $\left(\mathrm{dd}, J_{1}=8.4 \mathrm{~Hz}, J_{2}=7.8 \mathrm{~Hz}, 1 \mathrm{H}\right), 7.34(\mathrm{~m}, 1 \mathrm{H}) .{ }^{13} \mathrm{C} \mathrm{NMR}(151 \mathrm{MHz}, 1,1,2,2-$ tetrachloroethane- $\left.d_{2}, 100^{\circ} \mathrm{C}\right): \delta 139.95,134.19,133.95,130.04,130.00,128.10$, $126.83,124.74$ and 122.69 . (Two signals for the carbon atoms bonding to the boron atoms were not observed due to the quadrupolar relaxation of the boron atom); ${ }^{11} \mathrm{~B}-\mathrm{NMR}\left(128 \mathrm{MHz}, 1,1,2,2\right.$-tetrachloroethane- $\left.d_{2}, 100^{\circ} \mathrm{C}\right): \delta 69.3$; HRMS $(m / z):[\mathrm{M}]{ }^{+}$calcd for $\mathrm{C}_{40} \mathrm{H}_{24} \mathrm{~B}_{2} \mathrm{Br}_{2}$ : 686.0425; found, 686.0433 .

X-ray crystallographic analysis. Single crystals of $\mathbf{1}$ were obtained by slow diffusion of octane into a solution of $\mathbf{1}$ in chlorobenzene (Supplementary Fig. 11b). The intensity data were collected at $123 \mathrm{~K}$ with a Rigaku X-ray diffractometer equipped with a molybdenum FR-X microfocus generator, VariMax-Mo optics and a PILATUS $200 \mathrm{~K}$ detector. In total 22,714 reflections were collected, among which 5,303 reflections were independent $\left(R_{\text {int }}=0.0410\right)$. The structure was solved by direct methods (SHELXS-2013) and refined by full-matrix least-squares on $F^{2}$ for all reflections (SHELXL-2013). All non-hydrogen atoms were refined anisotropically and all hydrogen atoms were placed using AFIX instructions. The crystal data are as follows: Formula $\mathrm{C}_{40} \mathrm{H}_{24} \mathrm{~B}_{2} \mathrm{Br}_{2} ; \mathrm{FW}=686.03$, monoclinic, crystal size $0.14 \times 0.02 \times 0.02 \mathrm{~mm}^{3}, C 2 / c, a=19.259(7) \AA, b=17.907(6) \AA, c=18.056(6)$ $\AA, \beta=102.721(4) \AA, V=6,074(4) \AA^{3}, Z=8, D_{\text {calcd }}=1.500 \mathrm{~g} \mathrm{~cm}^{-3} ; R_{1}=0.0396$ $(I>2 \sigma(I)), w R_{2}=0.1006$ (all data), GOF $=0.987$

Thermogravimetric analysis. Thermogravimetric analysis was performed on a Seiko EXSTAR 6,000 TG/DTA 6,200 apparatus at a heating rate of $5^{\circ} \mathrm{C} \mathrm{min}-1$ under a nitrogen atmosphere (Supplementary Fig. 11c).

Characterization of precursor molecule. NMR spectra of the precursor $\mathbf{1}$ are shown in Supplementary Fig. $11 \mathrm{~d} .{ }^{1} \mathrm{H}$ and ${ }^{13} \mathrm{C}$ NMR spectra were recorded with a JEOL A-600 spectrometer $\left(600 \mathrm{MHz}\right.$ for ${ }^{1} \mathrm{H}$ and $151 \mathrm{MHz}$ for $\left.{ }^{13} \mathrm{C}\right) .{ }^{11} \mathrm{~B}-\mathrm{NMR}$ spectrum was recorded with a JEOL AL-400 spectrometer $\left(128 \mathrm{MHz}\right.$ for $\left.{ }^{11} \mathrm{~B}\right)$. Chemical shifts are reported in $\delta \mathrm{ppm}$ with reference to residual protons and carbons of 1,1,2,2-tetrachloroethane- $d_{2}\left(\delta 6.00 \mathrm{ppm}\right.$ in ${ }^{1} \mathrm{H}-\mathrm{NMR}$ and $\delta 73.78 \mathrm{ppm}$ in ${ }^{13} \mathrm{C}$ NMR). The external standard of $\mathrm{BF}_{3} \cdot \mathrm{OEt}_{2}$ was used for ${ }^{11} \mathrm{~B} \mathrm{NMR}$. The mass spectrum was measured with a Bruker Daltonics micrOTOF Focus using a positive-mode Atmospheric Pressure Chemical Ionization (APCI)-time of flight method in a $\mathrm{CHCl}_{3}$ solution. Thin layer chromatography was performed on plates coated with 0.25 -mm thickness of silica gel $60 F_{254}$ (Merck). Column chromatography was performed using silica gel PSQ 100B (Fuji Silysia Chemical).

Theoretical calculation. All first principles calculations in this work were performed using the periodic plane-wave basis Vienna ab-initio simulation package (VASP) code ${ }^{39,40}$ implementing the spin-polarized DFT. To accurately include van der Waals interactions in this system we used the optB86P functional ${ }^{41-43}$. Projected augmented wave potentials were used to describe the core electrons ${ }^{44}$. A kinetic energy cutoff of $550 \mathrm{eV}$ was found to converge the total energy of all the systems to within $10 \mathrm{meV}$. All STM simulations were done using the basic TersoffHamann approach ${ }^{45}$. The properties of the bulk and surface of $\mathrm{Au}$ and graphite, and a NO molecule, were carefully checked within this methodology, and excellent agreement was achieved with experiments. Systematic $k$-point convergence was checked for all systems and a $5 \times 1 \times 1$ mesh was found to be sufficient for the large GNR-Au systems. All reference calculations for adsorption energies, charge densities and projected density of states were performed with 4-anthracene GNR on gold, which had a lattice mismatch of only $1.4 \%$ (accommodated in the ribbon) When adding boron (B-GNR), this mismatch reduced to $0.3 \%$ as the ribbon expands slightly (Supplementary Fig. 4). To have a comparable anthracene separation of B-B sites as in experiments, particularly for the STM simulations, we also performed calculations on isolated 3-anthracene GNRs (for this size the mismatch with the Au surface would be over $10 \%$ unless an unfeasibly large unit cell is used). The simulated STM images were very similar, and hence were used for the image comparison in Fig. 1 for simplicity.

Calculated AFM image. Recently, several mechanical AFM models have been proposed $^{46,47}$, where the approach of a CO-functionalized AFM tip over a substrate can be simulated To obtain an accurate $3 \mathrm{D}$ force map. Key to these models is that during approach they allow the CO molecule to tilt under influence of the forces it experiences. This tilting proved to be key to understand the sharp contrast observed in atomic bonds. Here we used the model developed by Hapala et al. ${ }^{46}$ and extended it by adding electrostatic interactions on top of the van der Waals interactions. The B-GNR structure was taken from the DFT simulations of B-GNR on $\mathrm{Au}$ and the charges were extracted from the DFT calculations via Bader analysis ${ }^{48}$. The mechanical AFM model relies on empirical Lennard-Jones parameters, which were taken from the Chemistry at HARvard Molecular Mechanics (CHARMM) force field ${ }^{49}$, with additional boron parameters ${ }^{50}$. All other parameters are the same as intended by Hapala et al., and the simulated AFM scan is performed at a resolution of $0.025 \AA$ (in all directions), with a force tolerance criterion of $4 \times 10^{-6} \mathrm{eV} \AA^{-1}$. The 3D force field is subsequently converted into a frequency shift image ${ }^{51}$, using the experimental parameters $\left(f=24.76 \mathrm{kHz}, A=53 \mathrm{pm}\right.$ and $\left.k=1,800 \mathrm{~N} \mathrm{~m}^{-1}\right)$. In Fig. $3 \mathrm{~d}$ the simulated AFM image for the approximate same height as in the experiments is shown (the height in the bottom left corner of the figure denotes the distance of the carbon of the CO-molecule to the plane going through the carbons of the B-GNR).

\section{References}

1. Iijima, S. Helical microtubules of graphitic carbon. Nature 354, 56-58 (1991)

2. Novoselov, K. S. et al. Electric field effect in atomically thin carbon films. Science 306, 666-669 (2004).

3. Zhang, Y., Zhang, D. \& Liu, C. Novel chemical sensor for cyanides: boron-doped carbon nanotubes. J. Phys. Chem. B 110, 4671-4674 (2006)

4. Dai, J., Yuan, J. \& Giannozzi, P. Gas adsorption on graphene doped with B, N, Al, and S: a theoretical study. Appl. Phys. Lett. 95, 232105 (2009).

5. Biel, B., Blase, X., Triozon, F. \& Roche, S. Anomalous doping effects on charge transport in graphene nanoribbons. Phys. Rev. Lett. 102, 096803 (2009). 
6. Terrones, H., Lv, R., Terrones, M. \& Dresselhaus, M. S. The role of defects and doping in 2D graphene sheets and 1D nanoribbons. Rep. Prog. Phys. 75, 062501 (2012).

7. Xing, M., Fang, W., Yang, X., Tian, B. \& Zhang, J. Highly-dispersed borondoped graphene nanoribbons with enhanced conductibility and photocatalysis. Chem. Commun. 50, 6637-6640 (2014).

8. Wu, Z. -S., Ren, W., Xu, L., Li, F. \& Cheng, H. -M. Doped graphene sheets as anode materials with superhigh rate and large capacity for lithium ion batteries. ACS Nano 5, 5463-5471 (2011).

9. Panchakarla, L. S. et al. Synthesis, structure, and properties of boron- and nitrogen-doped graphene. Adv. Mater. 21, 4726-7430 (2009).

10. Zhao, L. et al. Local atomic and electronic structure of boron chemical doping in monolayer graphene. Nano Lett. 13, 4659-4665 (2013).

11. Wang, H. et al. Synthesis of boron-doped graphene monolayers using the sole solid feedstock by chemical vapor deposition. Small 9, 1316-1320 (2013).

12. Tang, Y. -B. et al. Tunable band gaps and p-type transport properties of boron-doped graphenes by controllable ion doping using reactive microwave plasma. ACS Nano 6, 1970-1978 (2012).

13. Grill, L. et al. Nano-architectures by covalent assembly of molecular building blocks. Nat. Nanotechnol. 2, 687-691 (2007).

14. Cai, J. et al. Atomically precise bottom-up fabrication of graphene nanoribbons. Nature 466, 470-473 (2010).

15. Bronner, C. et al. Aligning the band gap of graphene nanoribbons by monomer doping. Angew. Chem. Int. Ed. 52, 4422-4425 (2013).

16. Cai, J. et al. Graphene nanoribbon heterojunctions. Nat. Nanotechnol. 9 , 896-900 (2014)

17. Jäkle, F. Advances in the synthesis of organoborane polymers for optical, electronic, and sensory applications. Chem. Rev. 110, 3985-4022 (2010)

18. Araneda, J. F., Neue, B. \& Piers, W. E. Enforced planarity: a strategy for stable boron-containing $\pi$-conjugated materials. Angew. Chem. Int. Ed. 51, 9977-9979 (2012)

19. Dou, C., Saito, S., Matsuo, K., Hisaki, I. \& Yamaguchi, S. A boron-containing $\mathrm{PAH}$ as a substructure of boron-doped graphene. Angew. Chem. Int. Ed. 51, 12206-12210 (2012)

20. Treier, M. et al. Surface-assisted cyclodehydrogenation provides a synthetic route towards easily processable and chemically tailored nanographenes. Nat. Chem. 3, 61-67 (2011).

21. Koch, M., Ample, F., Joachim, C. \& Grill, L. Voltage-dependent conductance of a single graphene nanoribbon. Nat. Nanotechnol. 7, 713-717 (2012).

22. Ruffieux, P. et al. Electronic structure of atomically precise graphene nanoribbons. ACS Nano 6, 6930-6935 (2012).

23. van der Lit, J. et al. Suppression of electron-vibron coupling in graphene nanoribbons contacted via a single atom. Nat. Commun. 4, 2023 (2013).

24. Gross, L., Mohn, F., Moll, N., Liljeroth, P. \& Meyer, G. The chemical structure of a molecule resolved by atomic force microscopy. Science 325, 1110-1114 (2009).

25. Moll, N., Gross, L., Mohn, F., Curioni, A. \& Meyer, G. A simple model of molecular imaging with noncontact atomic force microscopy. New. J. Phys. 14, 083023 (2012).

26. Gross, L. et al. Organic structure determination using atomic-resolution scanning probe microscopy. Nat. Chem. 2, 821-825 (2010).

27. Gross, L. et al. Bond-order discrimination by atomic force microscopy. Science 337, 1326-1329 (2012).

28. Kawai, S. et al. Obtaining detailed structural information about supramolecular systems on surfaces by combining high-resolution force microscopy with ab initio calculations. ACS Nano 7, 9098-9105 (2013).

29. Luo, X. et al. Predicting two-dimensional boron-carbon compounds by the global optimization method. J. Am. Chem. Soc. 133, 16285-16290 (2011).

30. Requist, R. et al. Kondo conductance across the smallest spin $1 / 2$ radical molecule. Proc. Natl Acad. Sci. USA 111, 69-74 (2014).

31. Chen, Y. -C. et al. Tuning the band gap of graphene nanoribbons synthesized from molecular precursors. ACS Nano 7, 6123-6128 (2013).

32. Biel, B., Triozon, F., Blase, X. \& Roche, S. Chemically induced mobility gaps in graphene nanoribbons: a route for upscaling device performances. Nano Lett. 9, 2725-2729 (2009).

33. Marconcini, P. et al. Atomistic boron-doped graphene field-effect transistors: a route toward unipolar characteristics. ACS Nano 6, 7942-7947 (2012).

34. Bennett, P. B. et al. Bottom-up graphene nanoribbon field-effect transistors. Appl. Phys. Lett. 103, 253114 (2013).

35. Giessibl, F. J. High-speed force sensor for force microscopy and profilometry utilizing a quartz tuning fork. Appl. Phys. Lett. 73, 3956 (1998).

36. Hoffend, C., Schödel, F., Bolte, M., Lerner, H. -W. \& Wagner, M. Boron-doped tri(9,10-anthrylene)s: synthesis, structural characterization, and optoelectronic properties. Chemistry 18, 15394-15405 (2012).
37. Nesterov, E. E., Zhu, Z. \& Swager, T. M. Conjugation enhancement of intramolecular exciton migration in poly(p-phenylene ethynylene)s. J. Am. Chem. Soc. 127, 10083-10088 (2005).

38. Bieller, S. et al. Bitopic bis- and tris(1-pyrazolyl)borate ligands: syntheses and structural characterization. Organometallics 23, 2107-2113 (2004).

39. Kresse, G. \& Furthmuller, J. Efficiency of ab-initio total energy calculations for metals and semiconductors using a plane-wave basis set. Comput. Mater. Sci. 6, 15-50 (1996).

40. Kresse, G. \& Furthmüller, J. Efficient iterative schemes for ab initio total-energy calculations using a plane-wave basis set. Phys. Rev. B 54, 11169-11186 (1996)

41. Gulans, A., Puska, M. J. \& Nieminen, R. M. Linear-scaling self-consistent implementation of the van der Waals density functional. Phys. Rev. B 79, 201105 (2009).

42. Klimev, S. J., Bowler, D. R. \& Michaelides, A. van der Waals density functionals applied to solids. Phys. Rev. B 83, 195131 (2011).

43. Björkman, T., Gulans, A., Krasheninnikov, A. \& Nieminen, R. van der Waals bonding in layered compounds from advanced density-functional. Firstprinciples calculations. Phys. Rev. Lett. 108, 235502 (2012).

44. Blöchl, P. E. Projector augmented-wave method. Phys. Rev. B 50, 17953-17979 (1994).

45. Hofer, W., Foster, A. \& Shluger, A. Theories of scanning probe microscopes at the atomic scale. Rev. Mod. Phys. 75, 1287-1331 (2003).

46. Hapala, P. et al. Mechanism of high-resolution STM/AFM imaging with functionalized tips. Phys. Rev. B 90, 085421 (2014).

47. Hämäläinen, S. K. et al. Intermolecular contrast in atomic force microscopy images without intermolecular bonds. Phys. Rev. Lett. 113, 186102 (2014).

48. Tang, W., Sanville, E. \& Henkelman, G. A grid-based Bader analysis algorithm without lattice bias. J. Phys. Condens. Matter 21, 084204 (2009).

49. Brooks, B. R. et al. CHARMM: The biomolecular simulation program. J. Comput. Chem. 30, 1545-1614 (2009).

50. Baowan, D. \& Hill, J. M. Nested boron nitride and carbon-boron nitride nanocones. IET Micro \& Nano Lett. 2, 46-49 (2007).

51. Welker, J., Illek, E. \& Giessibl, F. J. Analysis of force-deconvolution methods in frequency-modulations atomic force microscopy. Beilstein J. Nanotechnol. 3, 238-248 (2012).

\section{Acknowledgements}

This work was supported in part by the Japan Science and Technology Agency (JST) 'Precursory Research for Embryonic Science and Technology (PRESTO)' for a project of 'Molecular technology and creation of new functions' to S.K. and S.S, by JST 'Core Research for Evolutional Science and Technology (CREST)' for a project of 'Development of high-performance nanostructures for process integration' to S.Y. and by the Swiss National Science Foundation, by the NCCR 'Nanoscale Science' of the Swiss National Science Foundation. A.S.F. and P.S. have been supported by the Academy of Finland through its Centres of Excellence Program project no. 915804 and EU project PAMS (contract no. 610446) and acknowledge use of the CSC, Helsinki for computational resources.

\section{Author contributions}

S.K. conducted experiments and carried out data analysis. S.S., S.O. and S.Y. synthesized the precursor molecule. A.S.F. and P.S. conducted theoretical calculations. S.K., S.S., S.Y., A.S.F., P.S. and E.M. contributed to writing the manuscript. S.K., S.S. and E.M. planned the project.

\section{Additional information}

Supplementary Information accompanies this paper at http://www.nature.com/ naturecommunications

Competing financial interests: The authors declare no competing financial interests.

Reprints and permission information is available online at http://npg.nature.com/ reprintsandpermissions/

How to cite this article: Kawai, S. et al. Atomically controlled substitutional borondoping of graphene nanoribbons. Nat. Commun. 6:8098 doi: 10.1038/ncomms9098 (2015).

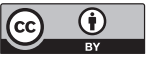

This work is licensed under a Creative Commons Attribution 4.0 International License. The images or other third party material in this article are included in the article's Creative Commons license, unless indicated otherwise in the credit line; if the material is not included under the Creative Commons license, users will need to obtain permission from the license holder to reproduce the material. To view a copy of this license, visit http://creativecommons.org/licenses/by/4.0/ 